\title{
AN ADAPTIVE NOTCH FILTER USING LMS ALGORITHM WITH VARIABLE STEP-SIZE FOR EEG SIGNALS
}

\author{
PHAM TRAN NHU ${ }^{1}$, HOANG MANH HA ${ }^{2}$ \\ ${ }^{1}$ Institute of Information Technology - VAST; ptnhu@ioit.ac.vn \\ ${ }^{2}$ College of BioMedical Instrumentations; hoangmanhha@yahoo.com
}

\begin{abstract}
This paper introduces an issue in designing a step size update for Least Mean Squared (LMS) algorithm to remove a transmission power line noise from noisy ElectroEncephaloGraphy (EEG) signals. We determine a sufficient condition for the convergence of a mentioned step-size updating for a LMS algorithm. A proposed adaptive filter is shown analytically to converge in the mean-square error sense. Simulation results illustrate that the performance of a new propose for step-size varying for the LMS algorithm is very effective.
\end{abstract}

Tóm tắt. Đối với lớp bài toán lọc nhiễu từ đường tải điện cho tín hiệu y sinh, bộ lọc triệt tần thích nghi với kích thước bước thay đổi là lựa chọn tốt nhất cho tốc độ hội tụ, độ ổn định và độ rộng dải triệt. Song, với trường hợp các tham số của nhiễu không còn thoả mãn các điều kiện hội tụ của thuật toán, ta cần có cách tính mới cho việc cập nhật kích thước bước thích nghi. Phân bố về độ lớn của gradient đã cung cấp ý tưởng và là cơ sờ cho phương pháp giải quyết bài toán. Bài báo đề xuất một phương pháp toán học trong việc sư dụng cách tính mới cho việc cập nhật kích thước bước thích nghi để lọc nhiễu trong quá trình ghi tín hiệu điện não đồ, xác định và chứng minh điều kiện đủ để thuật toán đề xuất là hội tụ.

\section{INTRODUCTION}

In the recording of biomedical signals, a contamination of power line interference is unavoidable. Moreover, the frequency of noise is not constant. So the elimination of the dynamic interferences caused by power transmission lines has been an interest research topic for the last few years. As we know, a fixed step size notch filter may eliminate the noise whose distribution is centered exactly at the filter frequency designed. However, the frequency of the power-line noise is not constant at exactly $50 \mathrm{~Hz}$. In cases of eeg signals recording, there are epileptiform oscillations with frequencies nearby the power line interference frequency which have been ignored because of the lack of an effective notch filter capable of eliminating the noise components distorted the original eeg signal. The situation suggests the need to design a notch filter with an optimal rejection bandwidth that effectively eliminates the time-varying noise caused by power. The problem was solved in [1] by adaptive notch filter under the assumption that noise frequency changes slowly. In the case when the noise frequency changes fast, adaptive notch filters with variable step sizes are used (see [2- -7]).

Choosing an adequate value of the step-size parameter will minimize the rejection band- 
width required to effectively eliminate the time-varying interference and will preserve optimal convergence, tracking and maladjustment conditions. The LMS algorithm with such a step size could avoid the cumbersome trial and error process.

We use an EEG database for evaluating practical designs in improving accuracy and minimizing complexity.

\section{AN ADJUSTMENT FOR STEP-SIZES OF LMS ALGORITHM}

\subsection{An adaptive notch filter using LMS algorithm with a fixed variable step-size parameter}

Magnitude of the transfer function of a fixed notch filter has a sharp shape (see Figure 1a).

The transfer function of an optimal notch filter is given by

$$
H(z)=\frac{1-2 \cos \left(2 \pi f_{0}\right) z^{-1}+z^{-2}}{1-2 \xi \cos \left(2 \pi f_{0}\right) z^{-1}+\xi^{2} z^{-2}},
$$

where, $\xi$ is a constant that defines the location of the poles in the unit circle; $f_{0}$ is a frequency of a power transmission noise.
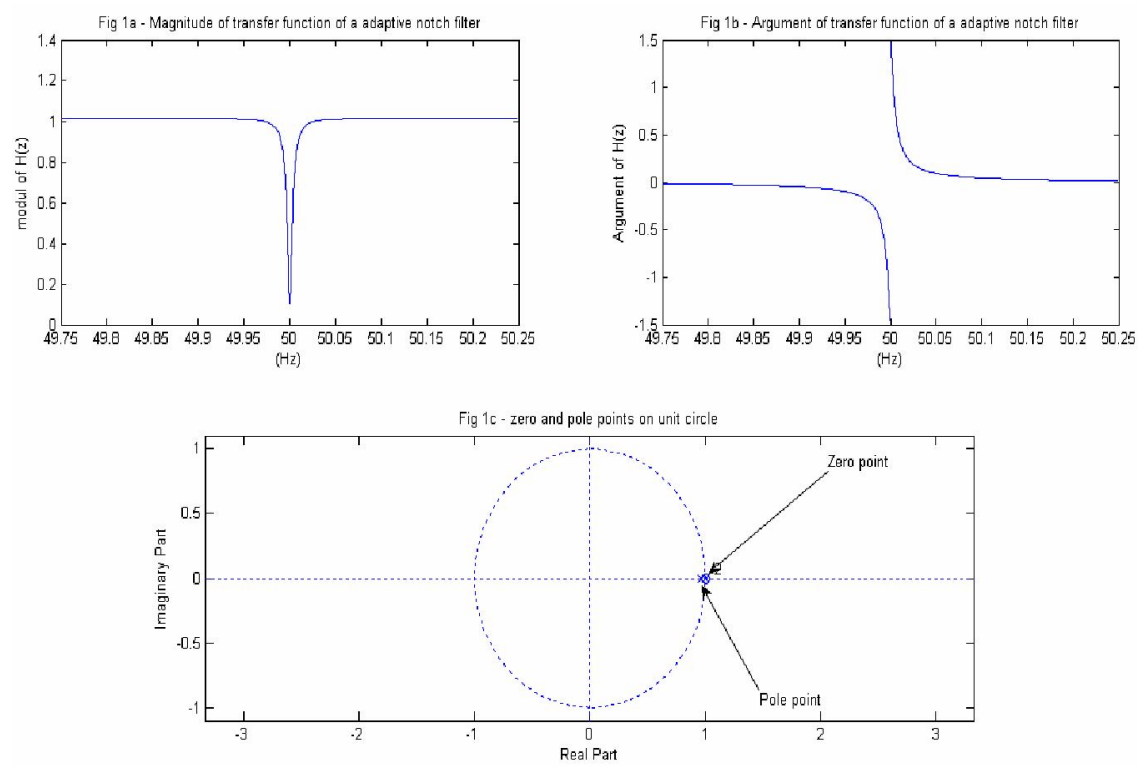

Figure 1. Magnitude of a transfer function of an optimal notch filter

A very narrow notch is usually desired in order to filter out a sinusoidal interference, which distorts the original signal. However, if the interference is not precisely known, and if the notch is very narrow, then the center of the notch may not fall exactly over the interference. When the reference for the interference is available, an adaptive noise canceling method originally proposed in [1] may be used. A central frequency of adaptive notch filter is adjusted for an error between it and frequency of noise to be minimal. B. We propose the following formulas 
for this adjustment.

$$
\begin{aligned}
& w_{1}(k+1)=w_{1}(k)+2 \mu \varepsilon(k) x_{1}(k), \\
& w_{2}(k+1)=w_{2}(k)+2 \mu \varepsilon(k) x_{2}(k),
\end{aligned}
$$

where $\mu$ - step-size parameter; $w_{1, k}$ and $w_{2, k^{-}}$weights of adaptive notch filter (see Figure 2 ).

$$
\begin{aligned}
& x_{1}(k)=C \cos \left(k 2 \pi f_{0}+\varphi\right), \\
& x_{2}(k)=C \sin \left(k 2 \pi f_{0}+\varphi\right) .
\end{aligned}
$$

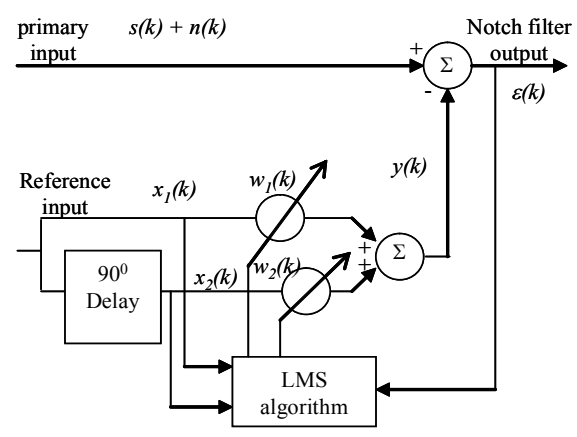

Figure 2. Model of noise cancel using an adaptive notch filter

The transfer function of the adaptive notch filter can be described as follows (see [1]).

$$
H(z)=\frac{1-2 z^{-1} \cos 2 \pi f_{0}+z^{-2}}{1-2\left(1-\mu C^{2}\right) z^{-1} \cos 2 \pi f_{0}+\left(1-2 \mu C^{2}\right) z^{-2}},
$$

where, $C$ - Amplitude of transmission power noise.

A bandwidth of ANF is determined by

$$
B W=\mu C^{2}
$$

\subsection{An adaptive notch filter using LMS algorithm with variable step-size param- eter}

The following step size updating was proposed in [2]

$$
\mu(k+1)=\alpha \mu(k)+\gamma \varepsilon^{2}(k),
$$

where, $\alpha$ - is a forgetting factor with values in $[0,1] ; \gamma$ - is a step size parameter for the adaptation of $\mu$.

Our motivation for updating step sizes is based on the rule the step size becomes smaller if the current $\left(w_{1}, w_{2}\right)$ is closer to the minimum point and becomes larger if it is far from minimum point. 
For a stability of the algorithm, the superior bound of all value step-size such that $\mu(k)<$ $\mu_{\max }$.

A rate of a step-size variation depend on factor $\gamma e^{2}(k)$. This solution is best for a determined distance from initial $\left(w_{1}, w_{2}\right)$ to minimum point.

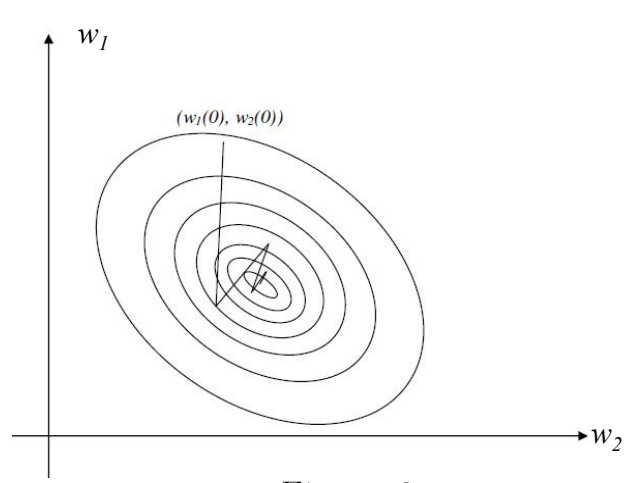

Figure 3

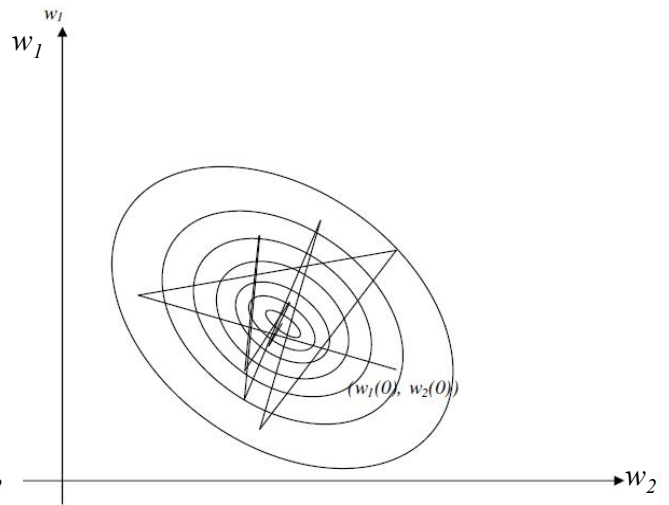

Figure 4

Figure 3: Method of LMS using variable step-size, with optimum start weight matrix (proposal in [2]).

The LMS algorithm with variable step-size, as writen about, requires an optimum initial weight matrix. The computational complexcity reflected in zigzag path in Figure 3.

The disadvantage of using formular (8) is that an initial weight matrix near minimum point randomly. In Figure 4, zigzag path reflects an increased computational complexcity and misadjustment of a LMS algorithm.

Figure 4: Method of LMS using variable step-size, with start weight matrix near a minimum point(proposal in [2]).

A positions of the minimum point is random, it denpend on random noisy signals. We need an another solution for any distance from initial weight matrix to minimum point.

To model the above idea, our work was suggested from a distribution of a magnitude of the gradient on $\left(w_{1}, w_{2}\right)$ plane (see Figure 5 )

$$
\mu(k+1)=\alpha\left|x_{1}(k) \varepsilon(k)\right|
$$

where, $\alpha\left|x_{1}(k) \varepsilon(k)\right|=|\nabla(k)|$.

However a magnitude of gradient $|\nabla|$ that is null at the minimum point on parapoloid (see [1]). It means that $\mu=0$ which can be substituted into the right hand side of Equation (7)

$$
B W=\mu C^{2}=0
$$

So bandwidth of ANF is null whenever the algorithm convergences and a ANF becomes a Band pass filter. To overcome this difficulty we introduce the step-size update rule as follows

$$
\mu(k+1)=\alpha\left|x_{1}(k) \varepsilon(k)\right|+\frac{\beta}{\left(\max \left\{x_{1}(k-n)|n=1, \ldots, 50|\right\}\right)^{2}},
$$


where, $\beta$ - an optimal value of a bandwidth of an ANF, $\left(\max \left\{x_{1}(k-n)|n=1, \ldots, 50|\right\}\right)^{2}$ returns current value of $C^{2}$.

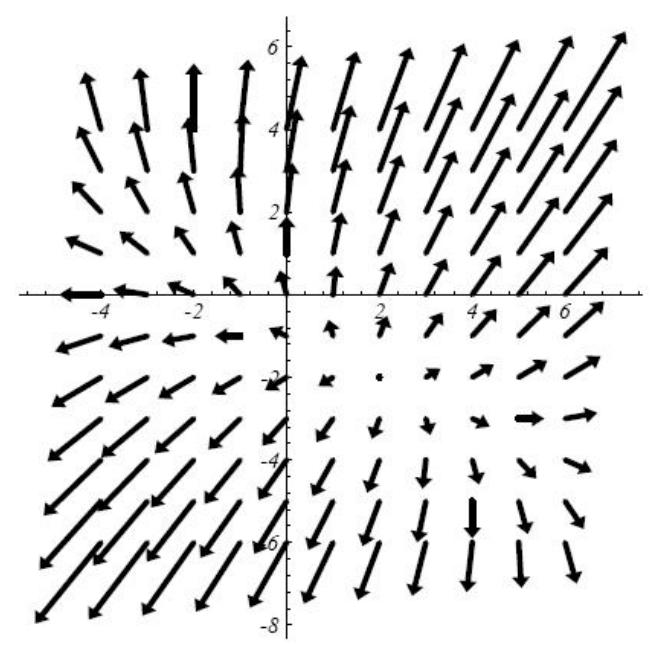

Figure 5. Gradient of the quadratic form

The last remark leads the second term of (11) to the constant form $\beta / C^{2}$.

\section{RESULT}

The following experiments have been carried out for the filter model $\{(2)$-(5), (11) $\}$ with $\mu=\mu(k+1), f_{0}=\frac{1}{N}, \varphi=0$. Here, $N$ is the number of samples over one period of statiscally stationary independent reference signals (4)-(5) with $E x_{i}^{2}(k)$ is the same for all $k, E x_{2}^{2}(k)$ equals to

$$
\frac{1}{N} C^{2} \sum_{k=1}^{N} \sin \frac{2 k \pi}{N} \sin \frac{2 k \pi}{N}=\frac{1}{N} C^{2}
$$

and $E x_{i}^{2}(k)$ equals to

$$
1-\frac{1}{N} C^{2} \sum_{k=1}^{N} \sin \frac{2 k \pi}{N} \sin \frac{2 k \pi}{N}=1-\frac{1}{N} C^{2} .
$$

The a sufficient condition for the convergence of mentioned filter model $\{(2)-(5),(11)\}$ is given by the following lemma

Lemma. If $\beta<1 / 2$ and $C<1$ then the sequences (2)-(3) of the mentioned filter model converge.

Proof. First of all, we consider the equation (2) with $\{(4),(11)\}$. From

$$
\varepsilon(k)=S(k)-\sum_{i=1}^{2} W_{i}(k) x_{i}(k),
$$


and $\{(2),(4),(11)\}$ follows the evaluation

$$
w_{1}(k+1)=w_{1}(k)+2 \mu(k+1) \varepsilon(k) x_{1}(k)=\left(1-2 \frac{\beta}{C^{2}} x_{1}^{2}(k)\right) w_{1}(k)+F_{k},
$$

where $F_{k}$ is the remaining parts of $\{(2),(4),(11)\}$ and its absolute value is bounded by some constant $M$ independent of $k$.

Convergence of the last equation follows from the estimation of the coefficient of the expected value $E v_{1}(k)$ of the linearzation system given by

$$
v_{1}(k+1)=\left(1-2 \frac{\beta}{C^{2}} x_{1}^{2}(k)\right) v_{1}(k) .
$$

The following estimation for $\left\{E v_{1}(k)\right\}$

$$
E v_{1}(k+1)=\prod_{l=1}^{k}\left[1-2 \frac{\beta}{C^{2}} E x_{1}^{2}(l)\right] E v_{0}=\left[1-2 \frac{\beta}{C^{2}}\left(1-\frac{1}{2} C^{2}\right)\right]^{k} E v_{0}=\left[1-\frac{\beta\left(2-C^{2}\right)}{C^{2}}\right]^{k} E v_{0}
$$

and the assumption $\beta<\frac{C^{2}}{\left(2-C^{2}\right)}$ yield the convergence of the linearization system. The convergence proof of $\left\{w_{2}(k)\right\}$ is the same on basis of the assumptions which ensures $\beta<1$ and then the coefficient of $E v_{2}(k)$ less than 1. We use EEG databases to evalue our method. The denoising of some EEG signals with serious $50 \mathrm{~Hz}$ noise. We applied the equation (12) below to compute a MSE. The rate of convergence of the MSE reflects the speed of a convergence of the algorithm and the midadjustment of MSE reflects stability of algorithm.

$$
M S E=\frac{1}{Q} \sum_{i=1}^{Q}|s(i)-\varepsilon(i)|^{2},
$$

where, $s(i)-N$ dimensional vector of the noise-free EEG signal; $\varepsilon(i)-N$ dimensional vector of the estimated EEG signal.

For the experiments presented in this section, $N=12000$ samples and $Q=200$ trials of the experiment.

\section{Experiment}

Figure 6 shows the MSE curves for three different cases of step-size parameter selection. For the first case the value of step-size parameter was fixed at $\mu=0.05$. This value is smaller than the optimum value found when using a variable step-size parameter and therefore the algorithm converges slowly after approximately 2,000 iterations. For the second case when the step-size parameter was fixed at $\mu=0.5$, near its maximum allowable value, we can observe that the algorithm converges very fast, after approximately 250 iterations, but has the disadvantage of a large misadjustment (more clearly at Figure 7).

The noisy EEG signal was also filtered by using our method for the variable step-size update. Clearly this method maintains equilibrium between fast convergence and small misadjustment. 


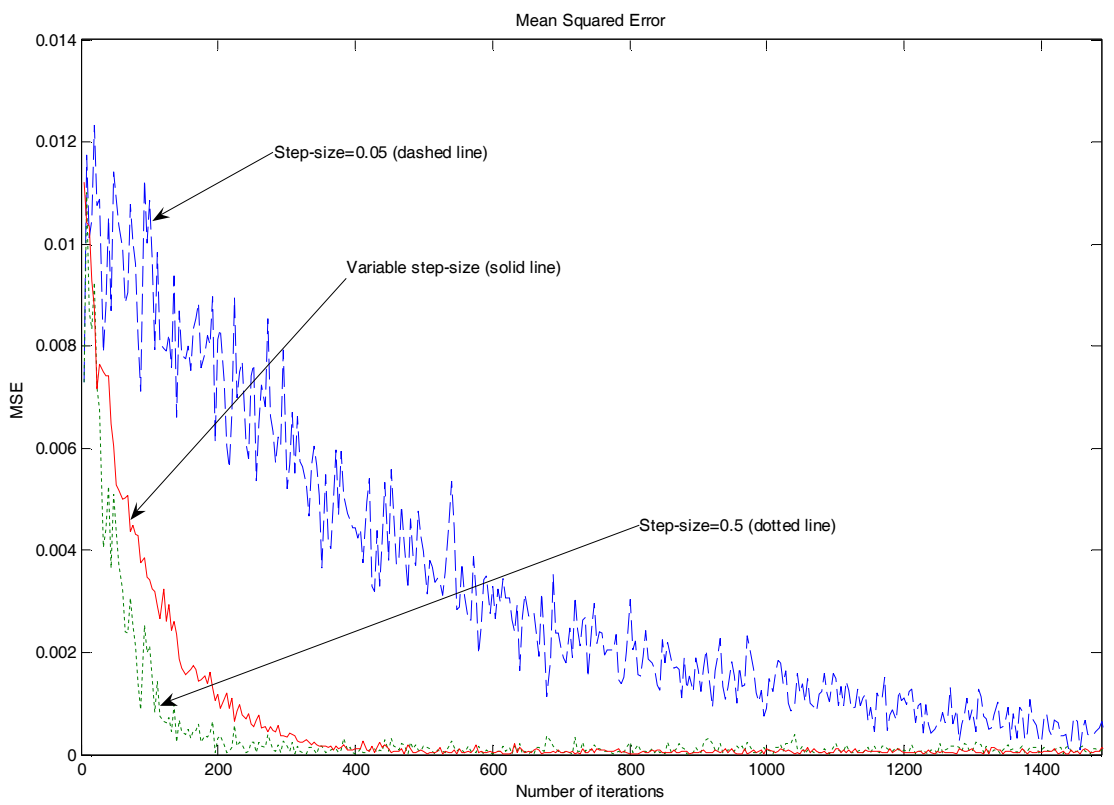

Figure 6. Comparison of MSE between LMS with a variable step-size parameter $\mu(n)$ and LMS with different fixed step-size parameters $\mu=0.05$, and $\mu=0.5$
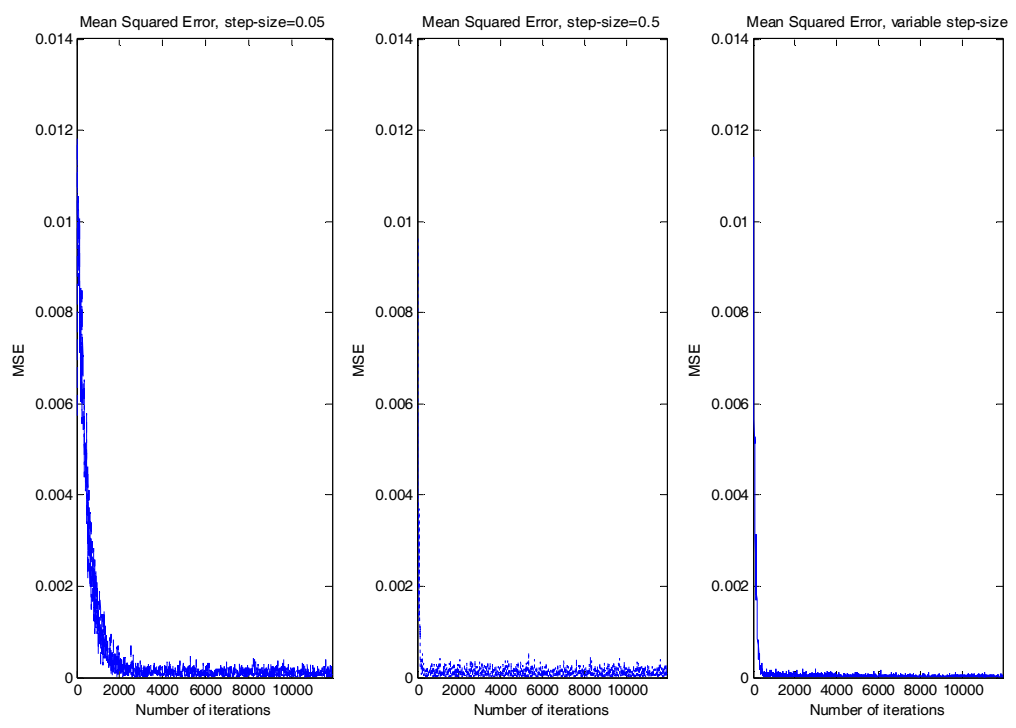

Figure 7. Comparison of MSE between LMS with a variable step-size parameter $\mu(n)$, and LMS with different fixed step-size parameters $\mu=0.05$, and $\mu=0.5$ (the misadjustment reflects stability)

Figure 8-11 show the noisy EEG signal, the original noise-free EEG signal, and the signal estimate $\varepsilon(n)$ obtained with the LMS algorithm using step-size fixed at $\mu=0.05$. 

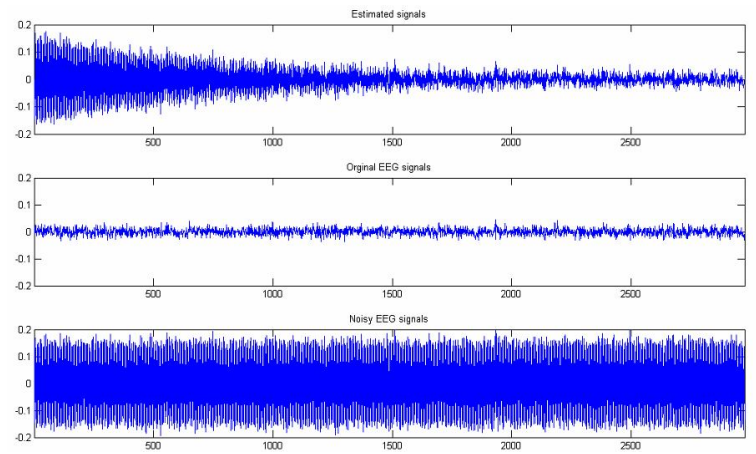

Figure 8
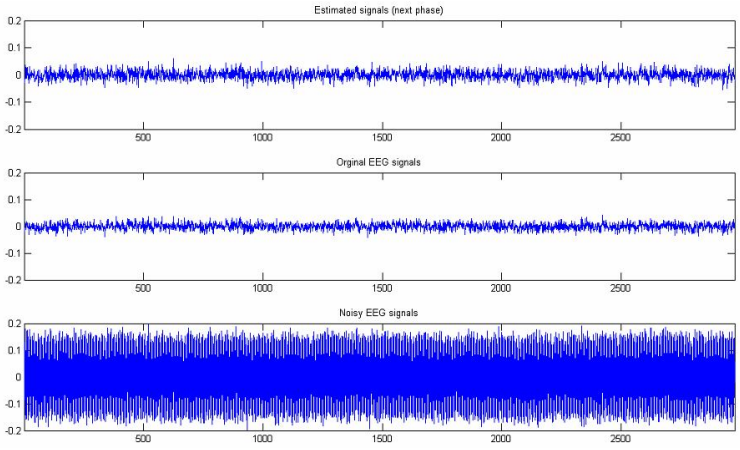

Figure 9

Figure 8: Estimated signal using fixed step-size $\mu=0.05$ (Top), Orginal Signal:(Center), Noisy EEG signal(Bottom).

Figure 9 (next phase): Estimated signal using fixed step-size $\mu=0.05$ (Top), Orginal Signal:(Center), Noisy EEG signal(Bottom).

By comparing these plots in the Figure 8, a remaining noise in estimated EEG signal is decreased slowly.
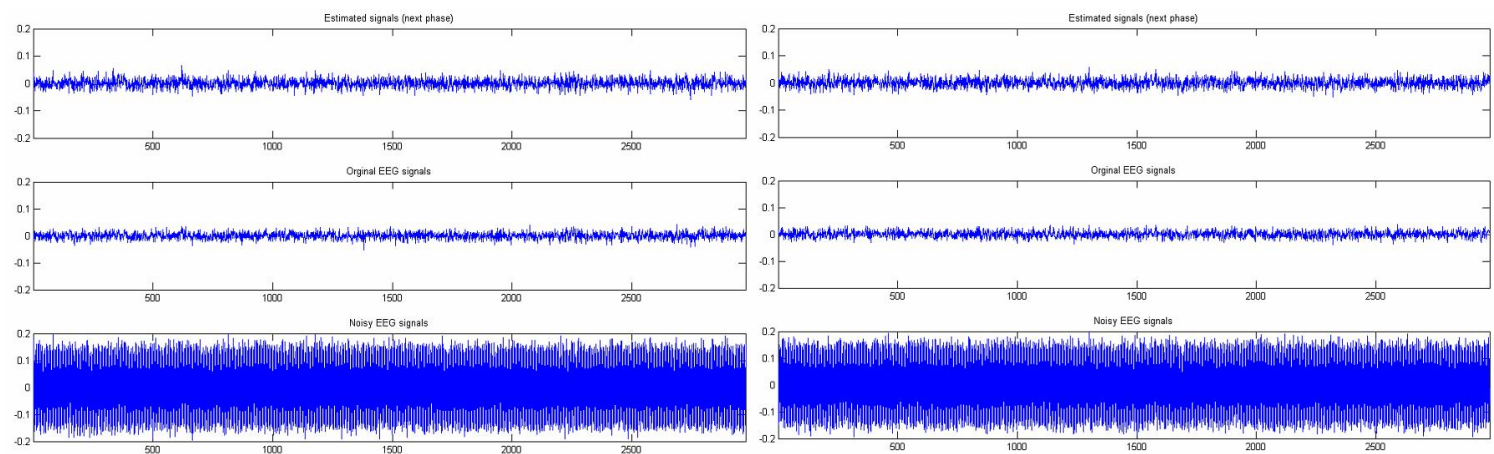

Figure 10

Figure 11

Figure 10 (next phase): Estimated signal using fixed step-size $\mu=0.05$ (Top), Orginal Signal: (Center), Noisy EEG signal(Bottom).

Figure 11 (next phase): Estimated signal using fixed step-size $\mu=0.05$ (Top), Orginal Signal:(Center), Noisy EEG signal(Bottom).

The results of the above denoising progress are shown continuesly in Figure 9, Figure 10 and Figure 11. An estimated EEG signals reflect that some noise remain in a denoised EEG signal.

In case of a step-size was kept constant with $\mu=0.5$. By comparing these plots in the Figure 12 a remaining noise in estimated EEG signal is decreased very fast. On the other hand, a rate of convergence will become faster when the step-size increases. 


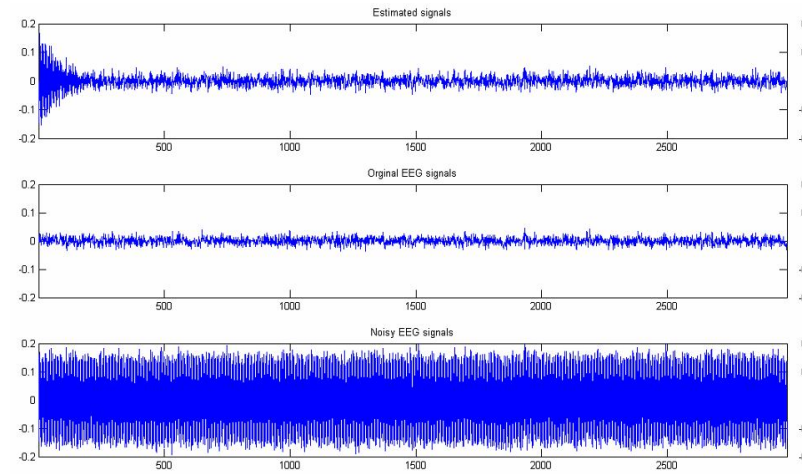

Figure 12

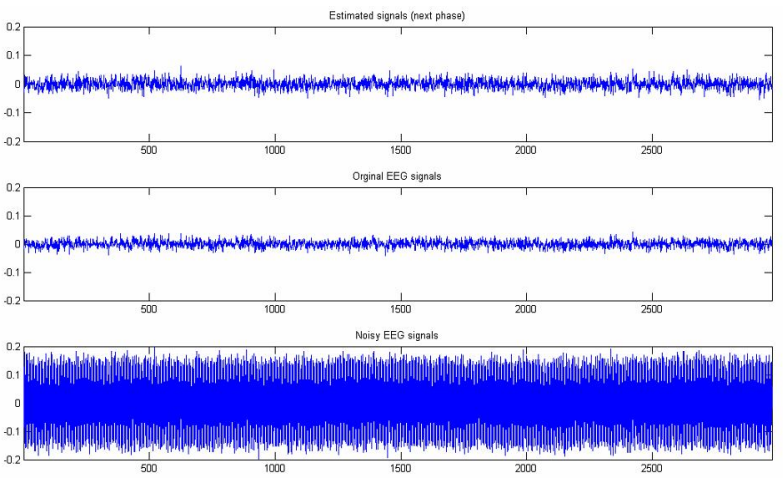

Figure 13

Figure 12: Estimated signal using fixed step-size $\mu=0.05$ (Top), Orginal Signal:(Center), Noisy EEG signal(Bottom).

Figure 13(next phase) : Estimated signal using fixed step-size $\mu=0.05$ (Top), Orginal Signal:(Center), Noisy EEG signal(Bottom).

A remaining noise can be informed by compairing the estimated EEG signals with orginal EEG signals. Following that way, a stability of algorithm will be decreases when step-size increase from 0.05 to 0.5 .

When a next phases was presented in Figure 13, Figure 14 and Figure 15, it is clearly that the noise could not remove perfectly if a step-size be fixed at big value.

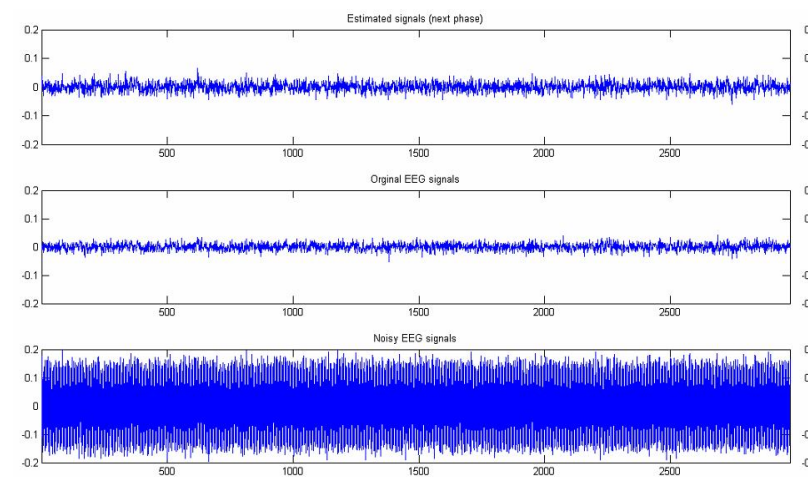

Figure 14

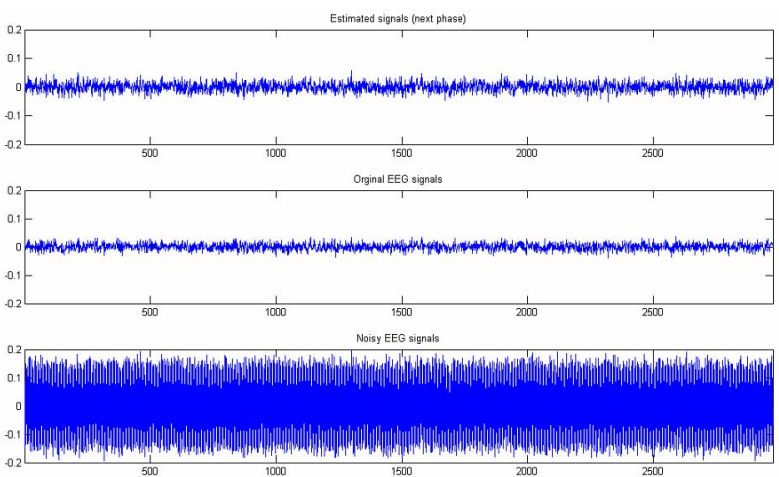

Figure 15

Figure 14 (next phase): Estimated signal using fixed step-size $\mu=0.05$ (Top), Orginal Signal: (Center), Noisy EEG signal (Bottom).

Figure 15 (next phase): Estimated signal using fixed step-size $\mu=0.05$ (Top), Orginal Signal:(Center), Noisy EEG signal (Bottom).

Figure ure 15 shows the EEG signal plus noise, the original noise-free EEG signal, and the signal estimate $\varepsilon(n)$ obtained with the varying step-size parameter algorithm. We can appreciate that the original signal is completely masked by the noise signal (at bottom of Figure 15 ), and how well it is reconstructed by the ANF system with varying step-size parameter 
proposed in this paper.

Figure 16 shows the ensemble average (over five hundred realizations) for the adaptation curve of the step-size parameter when using equation (11). The initial step-size parameter was set to $\mu_{0}=\mu_{\max }$ and this parameter converged to its average final value after approximately 300 iterations. Note that after the step-size has reached its average final value, which in this case was $\mu_{\text {final }}=0.005$, it continues to vary around this value.

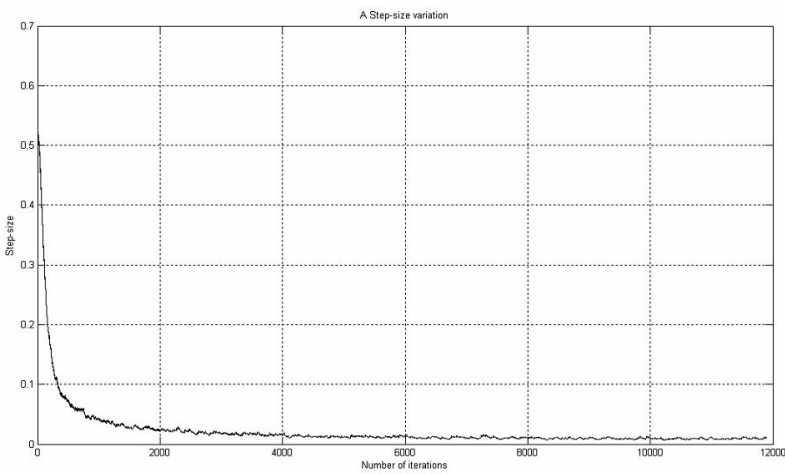

Figure 16

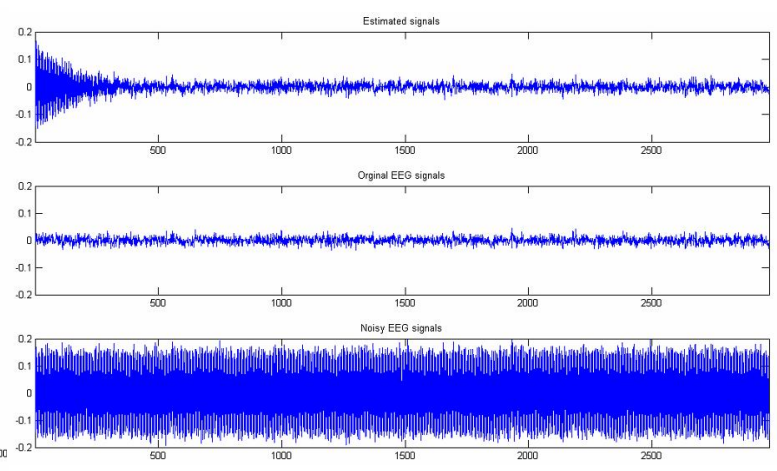

Figure 17

Figure 16: Convergence behavior of the step-size parameter.

Fig 17: Estimated EEG signals using variable step-size: $\mu=0.05$ (Top), Orginal EEG Signal: (Center), Noisy EEG signal (Bottom) On the other hand, a denoising is implemented on replacing an orginal EEG signal by an estimated EEG signal.

By comparing these plots with the EEG signals in Figure 17, Figure 18, Figure 19 and Figure 20, we can see that when the step-size parameter is adequately chosen, after convergence there is no distortion of the filtered signal. The evaluation of a rate of the algorithm using variable step-size will be finished by compairing Figure 17 with Figure 12. In case of a step-size was kept constant with $\mu=0.5$, the rate is faster slightly than case of using variable step-size. This is still true for the evaluation by MSE in Figure 6 .
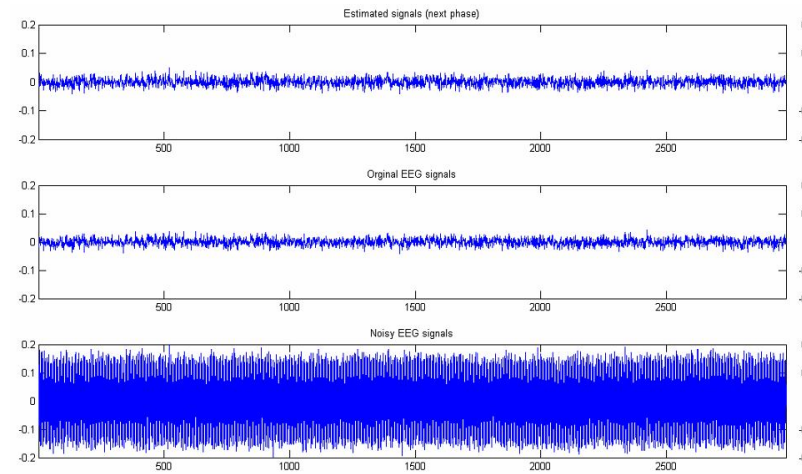

Figure 18
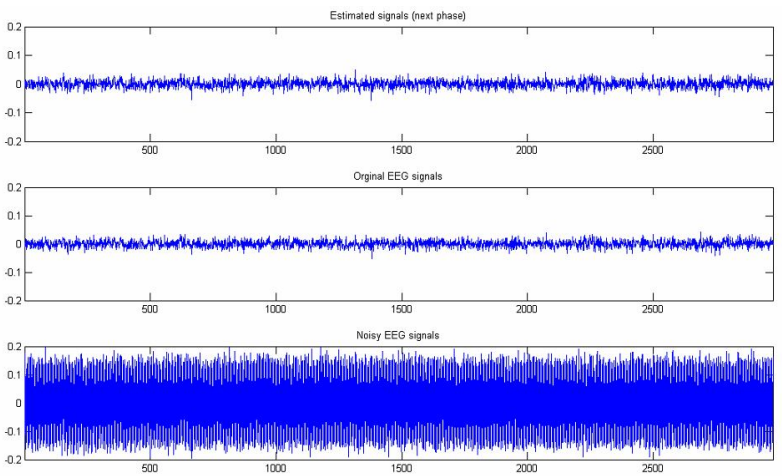

Figure 19

Figure 18: (next phase) Estimated signals using variable step-size: $\mu=0.05$ (Top) Orginal Signal: (Center), Noisy EEG signal (Bottom). 
Figure 19: (next phase) Estimated signals using variable step-size: $\mu=0.05$ (Top) Orginal Signal: (Center), Noisy EEG signal (Bottom).

Figure 6, Figure 16, Figure 17, Figure 18, Figure 19, Figure 20 reflect that the algorithm converges fast and stable. This is a optimum combination for Adative Noise canceler using LMS algorithm with step-size update.

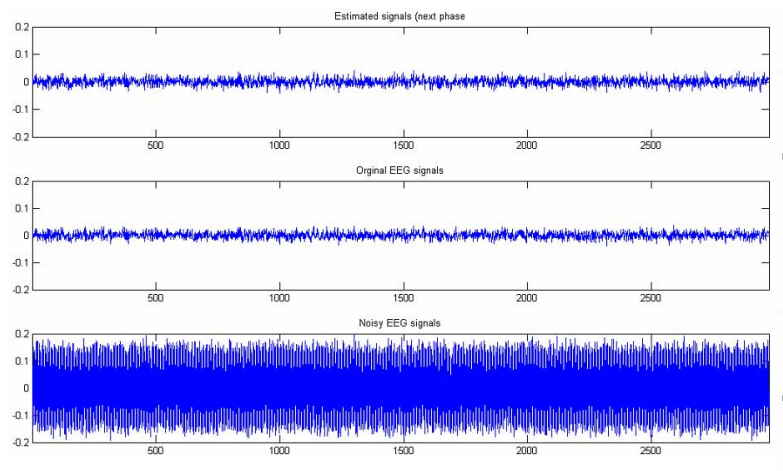

Figure 20

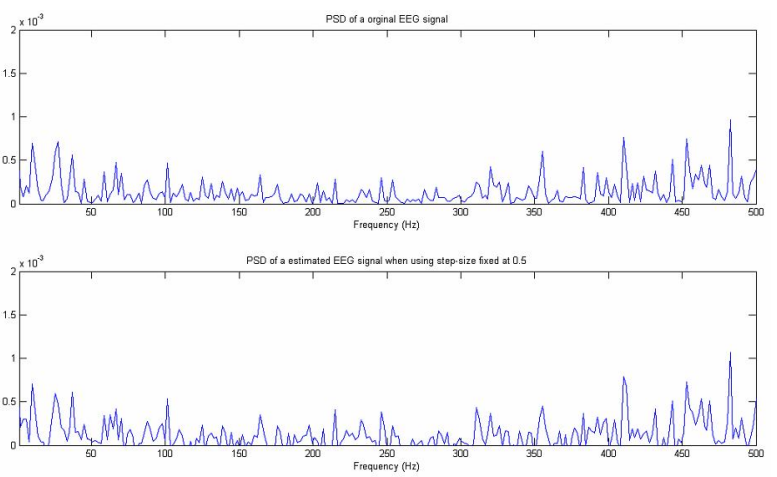

Figure 21

Figure 20: (next phase) Estimated signals using variable step-size: $\mu=0.05$ (Top) Orginal Signal:(Center), Noisy EEG signal(Bottom).

Fig 21: Comparison of power spectrum of a orginal EEG signal $\mu=0.05$ (Top) and Spectrum of a estimated EEG signal(bottom) when using fixed step-size $\mu=0.5$.

There is a distortion of the spectral content in the filtered signal when using fixed step-size $\mu=0.5$ (see Figure 21).
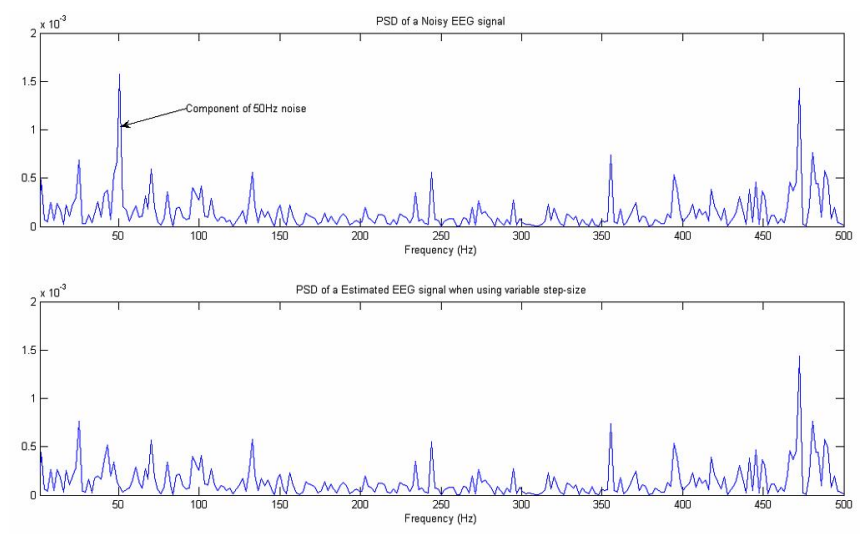

Figure 22. Comparison of power spectrum of a noisy EEG signal and spectrum of a estimated EEG signal when using variable step-size

Figure 22 shows the Power spectrum density of the noisy EEG signal (top) and the estimated EEG signal (bottom) when using the variable step-size in this experiment. By comparing these plots we can see that when the step-size parameter is adequately chosen, there is no distortion of the spectral content in the filtered signal. Component of $50 \mathrm{~Hz}$ noise is removed only. 
Comparision with proposal in [2]

For the case the step-size parameter adjustment using Equation (8) the algorithm converged after approximately 500 iterations [2]. For the case the step-size parameter adjustment using Equation (11), we can observe that the algorithm converged after approximately 300 iterations.

Based on the result of column (2) in this table, we compute band width of the filter use formular (7). When formular (11) is used we expect that well over $90 \%$ of the band width will be narrowed. It means that using formular (11) is able to improve an acuracy of the LMS algorithm in a remove a power transmittion noise problem. It could avoid a removement of epileptiform oscillations with frequencies nearby the power line interference frequency which have been ignored because of the lack of an effective notch filter not narrow enought.

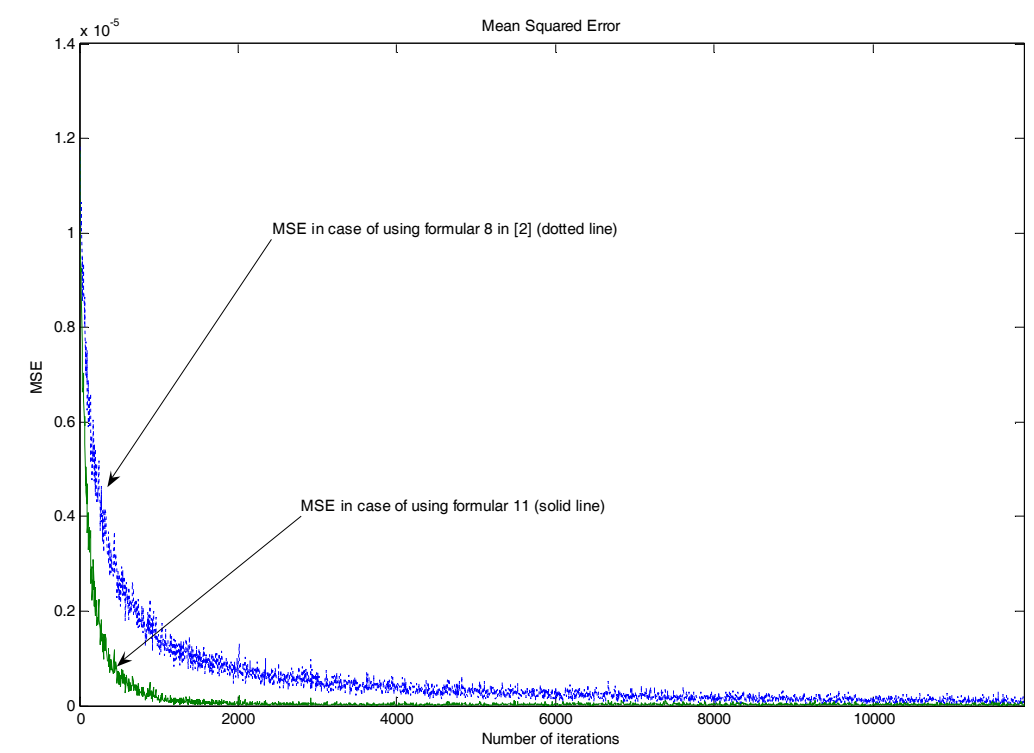

Figure 23. Comparison of MSE using formular (8) (dotted line) and using formular (11) (solid line)

Table 1. Performance of the measure when the step-size variation with our propose and with formular (8) in [2]

\begin{tabular}{|l|l|l|l|}
\hline \multicolumn{1}{|c|}{ Mesure } & $\begin{array}{c}\text { An average optimum } \\
\text { value of Step-size }\end{array}$ & Band width & $\begin{array}{c}\text { Number of iterations to } \\
\text { converge }\end{array}$ \\
\hline \multicolumn{1}{|c|}{$(1)$} & \multicolumn{1}{|c|}{$(2)$} & $(3)$ & \multicolumn{1}{c|}{$(4)$} \\
\hline $\begin{array}{l}\text { proposed variable } \\
\text { step-size }\end{array}$ & 0.005 & 0.0001125 & 300 \\
\hline Variable step-size [2] & 0.05 & 0.0011 & 500 \\
\hline
\end{tabular}

A number of iterations for convergence is approximate 500 iterations when LMS algorithm use formular (8) to vary a step-size [2], during number of iterations for convergence is approximate 300 iterations when LMS algorithm use formular (11) to vary a step-size.

In Figure 24, the begins at $\left(w_{1}(0), w_{2}(0)\right)$, the same point with start point in Figure 4, and stops at the minimum point. The zigzag path reflects that the number of iterations can 
be reduced by using formular (11).

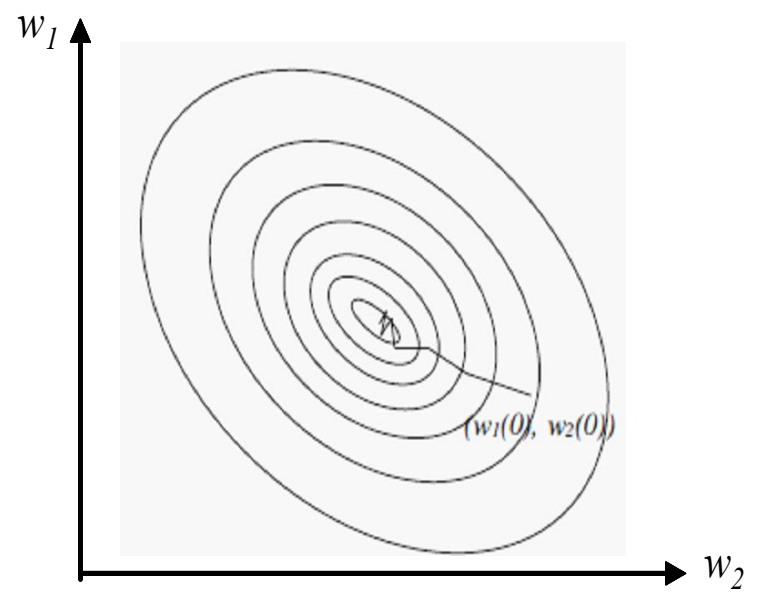

Figure 24. Method of LMS using variable step-size, with optimum start weight matrix (our proposal [2])

\section{CONCLUSION}

For the three different cases of step-size parameter selection we can readily identify the instants in which the noise drifted in frequency. It is important to notice that in all cases the filters were able to track the frequency changes. However, the variable step-size algorithm was able to track the frequency changes while maintaining a fast convergence rate, a small misadjustment and an optimum step-size value.

An adaptive noise canceler system based on a variable step-size LMS algorithm is able to find an optimum speed of convergence which is of great importance in real-time applications and allows the minimization of information loss and signal distortion. The proposed filters could be implemented in existing EEG recording devices or in new devices intended for realtime ambulatory EEG monitoring.

The choice of the step-size parameter in the adaptation algorithm plays an important role in combination of the rate of convergence, stability, tracking capabilities and rejection bandwidth of the filters. The proposed variable step-size method may overcome the cumbersome trial and error process needed to choose an adequate value for parameters and will minimize the rejection bandwidth required to effectively eliminate the timevarying interference introduced by power transmission lines. This last property is of great importance since, as mentioned in the introductory paragraphs, valuable signal information is found around the interference frequency band.

\section{REFERENCES}

[1] B. Widrow, Samuel D. Stearns, Adaptive Signal Processing, Prentice-Hall, 1985.

[2] D.O. Olguin, F. Bouchereau, S. Martinez, Adaptive notch filter for EEG signals based on the LMS algorithm with variable step-size parameter, Conference on Information Sciences and 
Systems, The Johns Hopkins University, March 16-18, 2005.

[3] Koike S, A class of adaptive step-size control algorithm for adaptive filter, IEEE Trans, Signal Process 50 (6) (2002) 1315-1326.

[4] Koike S, Convergence analysis of adaptive filters using normalized sign-sign algorithm, IEICE Trans. on Fundamental of Electronics, Communications and Computer Sciences bf Vol E88-A (11) (2005) 3218-3224.

[5] Koike S, Stability conditions for adaptive algorithms with non-quadratic error criteria, EUSIPCO2000, Tampere, Finland, Sep., 2000.

[6] K. W. Chan, Y.T. Zhang, Noise reduction of motion artifact from photoplethysmographic recording using a variable step-size LMS filter, Proceeding of IEEE 2 (2002) 1343-1346.

[7] Hoang Manh Ha, Variable step size LMS filter for ecg signal, Proceedings of the International Conference on BioMedical Engineering, The Hanoi University of Technology, July 27-29, 2007.

Received on October 14 - 2003

Revised on January 14 - 2004 